\title{
Dominancia minoritaria: dos experimentos exploratorios
}

\author{
ELENA GAVIRIA, JESÚS SOLANO, MERCEDES CARMONA \\ y JOSÉ MIGUEL FERNÁNDEZ DOLS . \\ Universidad Autónoma de Madrid
}

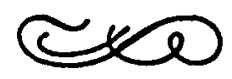

Resumen

A partir de los experimentos de Asch, el estudio de la relación entre mayoría y minoria ha sido estudiado en términos del ajuste o desviación con respecto a la norma. En este artículo se propone una nueva perspectiva interactiva: el grupo es una microestructura social en la que los individuos se enfrentan entre si dando lugar a to que los etólogos denominan srelaciones de dominancias. En los experimentos expuestos los resultados muestran una dominancia de la minoría que no guarda relación directa con los procesos de infuencia mayoritaria o minoritaria.

\section{Abstract}

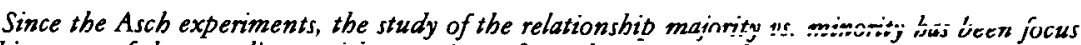
ed ${ }^{\prime}$ terms of the unnitiance with or deviance from the norm. In this paper a interactional point of view for the study of the group is proposed; the group, such as is posed in Asch paradigm, would be a social microestructure in which individuals struggle one with each other. It is, thus, an asymetric interaction that may be understood in terms of adominance relationshipsw. In two experiments a Reversed-Treatment Control Group Design with Pretest and Posttest was employed; the experimental treatment was a modified version of Asch paradigm. Results support an affective and behavioral dominance of minority that has not a direct relationship with influence processes.

Dirección del autor: Dto. de Psicología Básica, Social y Metodología. Facultad de Psicología, Universidad Autónoma de Madrid, 28049 Madrid.

Agradecimientos: Parte de la financiación de este trabajo se debe al proyecto, financiado por CAICyT PB85-0226. 


\section{INTRODUCCION}

En la investigación sobre influencia y conformidad uno de los temas más oscuros de la investigación actual es la caracterización conductual y afectiva de mayorías y minorías. Moscovici (1985), en una reciente revisión, señala la necesidad de estudiar la obvia fascinación que produce la minoría, sea ésta un grupo $o$ un individuo.

La alternativa que exploraremos en el presente trabajo, consiste en considerar que la situación de Asch aplicada a un grupo pequeño provoca fundamentalmente una asimetría interactiva, un conflicto o desacuerdo en el que, debido a la corta vida del grupo experimental, no se generan normas propias.

En tal caso no podemos caracterizar a la minoría como desviada ya que no existen, pues, normas referenciales: nos encontramos ante una microestructura social abierta e inestable en la que los individuos se enfrentan unos a otros; el ganador impone sus criterios a los demás. Se trata, pues, de una situación que puede entenderse de acuerdo con lo que los etólogos denominan relaciones de dominancia social. Tal dominancia aparecería aquí en una versión muy elemental incluso comparándola con las estructuras de poder de los primates no humanos (vid. por ej. De Wall, 1982) y podría, por otra parte, considerarse como un estado embrionario de las relaciones de liderazgo que exigen la existencia de roles establecidos (Shaw, 1976).

Además, el paradigma de Asch tiene un valor especial cara a estudiar la conducta dominante puesto que sitúa a un individuo contra el resto del grupo. Según esto, si se produce dominancia será suficiente manipular aquellas variables consideradas por la literatura como más relevantes en la emergencia de dominancia para producir tal fenómeno en el laboratorio.

El concepto de dominancia es excesivamente amplio para dedicarle en este informe experimental una revisión completa. Los investigadores han propuesto diversos criterios como índices de dominancia, según la orientación teórica y las especies estudiadas. Algunos criterios se refieren al individuo cuya conducta es un signo de dominancia mientras que otros se centran en la conducta de los subordinados hacia el sujeto dominante. Entre los criterios más citados se encuentra la prioridad de acceso a incentivos, «ser el ganadors en enfrentamientos agonísticos, recibir conductas de sumisión o contacto (grooming) y ser objeto de la atención de los demás miembros del grupo. Además, se han identificado varios factores como determinantes de dominancia, tales como relaciones de consanguineidad, alianzas interindividuales, o ciertas características individuales tales como edad, sexo o corpulencia (vid., por ejemplo, Rowell, 1974; Richards, 1974; Peláez, 1985 como revisiones del concepto de dominancia. También, como ejemplos de estudios etológicos de dominancia en humanos Strayer y Strayer, 1976; Sluckin y Smith, 1977; Gottman y Ringland, 1981).

Un fenómeno que ha sido considerado tanto índice de dominancia como determinante de ella ha sido la atracción que ciertos individuos ejercen sobre el resto del grupo. El modelo de Estructura Social de la Atención de Chance (1967) está basado en dicha atracción; de acuerdo con el modelo el individuo dominante es el que recibe el mayor monto de atención por parte de los otros miembros del grupo. Este individuo poseería alguna característica concreta que lo distinguiría de los demás y lo convertiría en el objeto de la atención del grupo (vid. también, Chance y Larsen, 1976). 
A partir de dicha observación y de nuestro trabajo previo (Fdez. Dols, 1981) una hipótesis que debe explorarse es que, con independencia de los procesos de influencia social, el pertenecer a la minoria numérica implique ciertas ventajas posicionales que puedan facilitar su dominancia sobre el resto del grupo.

El estudio de la emergencia de la conducta dominante implica que todo el grupo actúa espontáneamente (no hay cómplices del experimentador) y de hecho interactúan (es decir, hay una tarea de grupo y no una simple manifestación de juicios). Según esto, necesitamos estudiar las relaciones de dominancia que se producen en situaciones semejantes a las de Asch pero con las siguientes modificaciones:

a) todos los sujetos deben ignorar, a lo largo de la sesión experimental, que son víctimas de un tratamiento que les asigna un rol mayoritario o uno minoritario en el grupo.

b) los sujetos no deben haber interactuado previamente, haciéndolo por primera vez en la sesión experimental. En nuestro diseño la sesión experimental se dividirá en tres partes: unos minutos de interacción previos a la manipulación experimental, la manipulación experimental propiamente dicha (que determina una mayoría y una minoría) y, tras dicha situación, un período final de interacción posterior al tratamiento. En la notación de Campbell y Stanley (1966), el esquema del diseño sería «O X O».

A continuación presentamos dos series experimentales destinadas a corroborar nuestra hipótesis.

\section{METODOLOGIA GENERAL DE LOS EXPERIMENTOS}

Para esta descripción seguiremos, en parte, la más detallada de FernándezDols (1084).

El procedimiento experimental indicado consta, como se señaló anteriormente, de tres etapas que tienen lugar en una sola sesión experimental.

Dicha sesión transcurre de acuerdo con el siguiente guión: se cita por separado a cuatro sujetos y, cuando éstos llegan al laboratorio, un experimentador (que se retira inmediatamente después) les indica que deben realizar conjuntamente una tarea manual sobre una mesa sin sillas (resolver un puzzle o jugar con una arquitectura de juguete) durante un período de tiempo no especificado. Cuando los sujetos han resuelto el puzzle o acabado el juego, deben avisar al experimentador que durante este tiempo ha permanecido sentado en un rincón de la sala, de cara a la pared, aparentemente absorto en la lectura y sin interactuar postural o visualmente con el grupo.

A continuación el experimentador les solicita que se sienten en cuatro mesas en hilera y separadas entre sí por pantallas, para iniciar el tratamiento experimental propiamente dicho.

Dicho tratamiento es una versión abreviada y modificada del experimento de Asch (Fernández-Dols, 1981) que permite controlar simultáneamente tres variables (mayoría o minoría numérica; atribución de competencia y ambigüedad de la condición perceptiva) sin necesidad de cómplices del experimentador.

Para lograr la ambigüedad o claridad de la situación perceptiva (grado de incertidumbre) se utilizó un estímulo muy sencillo proveniente de los trabajos 
de Frenkel-Brunswik (1948) sobre prejuicio y tolerancia a la ambigüedad en niños. El reactivo utilizado por Frenkel-Brunswik es un gato que paulatinamente, a lo largo de siete viñetas pierde sus rasgos característicos, pasa por una serie de viñetas ambiguas (viñetas 3, 4 y 5 ) y finalmente se convierte en un perro (viñeta 7).

Utilizando estos reactivos se elaboraron una serie de tarjetas que mostraban una de las figuras de la escala gráfica de Frenkel-Brunswik. La tarea que se solicitaba a los sujetos era indicar cuantos perros o gatos habían visto tras mostrárseles secuencialmente un número determinado de cartas.

La claridad o ambigüedad del reactivo se logró fácilmente según que los perros o gatos mostrados en las tarjetas fueran «claros» (esto es, pertenecientes a las viñetas o grados 1, 2, 6 y 7) o «ambiguos» (esto es, pertenecientes a las categorías 3,4 y 5 de la escala de Frenkel-Brunswik).

La pertenencia a la mayoría o la minoría numérica se decidía también fácilmente sin necesidad de cómplices. Las tarjetas mostradas tenían un anverso y un reverso con diferentes figuras. Según la serie de caras mostradas los sujetos veían una diferente combinación de figuras. De este modo es fácil provocar la emergencia de un juicio mayoritario mostrando a todos los sujetos, con excepción de uno, la misma combinación de reactivos. El sujeto restante se convierte 'en la minoría cuando, sin darse cuenta de ello, debe observar una secuencia de cartas diferente a las de los demás, de tal modo que su juicio es, casi inevitablemente, divergente.

Con vistas a comparar la tendencia a interpretar un papel dominante en el grupo por parte de la mayoría y la minoría (con independencia de la influencia social manifestada durante la situación de conformidad) se manipuló la atribución de competencia. El experimentador indicaba como correctos, en las situaciones de divergencia, los juicios bien de la mayoría numérica o bien de la minoría.

Con respecto a nuestra facilitación de la dominancia en términos de competencia (vid. experimentos) podemos afirmar que la competencia es habitualmente la característica más importante relacionada con dominancia en un grupo orientado a la tarea, aunque indudablemente pueden haber otros rasgos importantes, tales como por ejemplo apariencia física. En nuestro caso los grupos se diseñaton orientados a una tarea, como se indicará en su momento.

En todos los experimentos que comentamos a continuación se utilizaron cuatro series de tarjetas. La primera, una serie de reactivos idéntica para todos los sujetos, con uno de categoría 1 , dos de la categoría 2 y tres de la categoría 7 . Una vez se habían mostrado las cartas a cada sujeto (uno a uno siempre), el experimentador se aseguraba de que todos los sujetos habían comprendido la tarea y que todos sus juicios eran idénticos; como se indicó anteriormente, lo que se solicitaba a los sujetos era identificar cuántas veces habían visto un perro (es decir, un reactivo de la categoría 7) en las series de tarjetas.

Tras esta primera presentación, el experimentador mostraba una segunda serie de tarjetas que, aparentemente, contenían un solo conjunto de figuras (una por tarjeta) pero que de hecho tenían además otro juego de reactivos al dorso. A la mayoría numérica (tres sujetos) se les presentaban cinco reactivos de la categoría 1 y tres de la categoría 7; el sujeto minoritario observaba el dorso de las tarjetas con cuatro reactivos de la categoría 1 y otros cuatro de la 7.

Esta presentación, destinada a provocar disidencia entre la mayoría y la minoría, era seguida por una tercera serie con el mismo propósito e idéntica me- 
cánica (siete reactivos del 7 y cinco del 1 para la mayoría y seis de cada para la minoría). Por último se presentaba una cuarta serie de tarjetas con distintas características según el experimento (vid. Fdez. Dols et al. 1984).

Al sujeto se le pedía que indicara cuántos perros (reactivos de la categoría 7) había visto. Tras la segunda y tercera series (i. e. en situaciones de divergencia) el experimentador, oídos los juicios de los sujetos, simulaba leer una hoja con la solución que coincidía (según el diseño) con el juicio de la mayoría o de la minoría.

Si en la cuarta serie (situación crítica) aparecía una situación de divergencia no se indicaba una solución "correcta» sino que el experimentador pedía a los sujetos repetir la tarea de acuerdo con la siguiente instrucción:

Veamos, sería bueno ponernos de acuerdo, vamos a intentarlo de nuevo...

Si la divergencia aparecía de nuevo, esto es, si la minoría no se plegaba hacia la mayoría o viceversa, se hacía una segunda repetición, finalizando así la situación. Si en algún momento de las series críticas se producía la confluencia el tratamiento se interrumpía finalizando la situación.

El resultado en términos de influencia mayoritaria o minoritaria es, pues, la confluencia o no confluencia, durante este tratamiento, de la mayoría hacia la minoría o viceversa.

Obtenida así la división del grupo en una mayoría y una minoría, el experimentador solicitaba a los sujetos que llevaran a cabo una segunda tarea, de características similares a la primera y en las mismas condiciones. Dicha situación se utilizaba, como se verá a continuación, para determinar ciertos indicadores de dominancia mayoritaria o minoritaria en términos interactivos y con independencia de los indicadores de influencia social (confluencia en los juicios perceptivos).

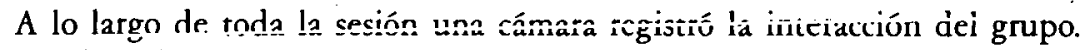

Tras la sesión se llevó a cabo una entrevista postexperimental en la que se solicitó a los sujetos que indicaran lo siguiente: (1) si conocía anteriormente a algún miembro del grupo (la respuesta fue negativa en todos los casos en los experimentos de este informe). (2) Que intentaran recordar los juicios expresados por cada miembro del grupo en la sesión experimental. (3) Que indicaran con cuál de las personas del grupo estarían dispuestos a hacer una tarea semejante. (4) Que comentaran la tarea del grupo y las posibles causas de la divergencia; esta última pregunta tenía sobre todo la finalidad de detectar si los sujetos sospechaban del engaño implicado en el tratamiento experimental (las respuestas de los sujetos en los experimentos relatados atribuyen las divergencias a diferencias en percepción y sólo cuatro sujetos parecen sospechar).

\section{EXPERIMENTO 1}

Como se indicó anteriormente, el experimento 1 intenta comprobar si una situación de conflicto-divergencia entre mayoría y minoría implica, si la situación lo permite, una pauta de comportamiento competitivo en la que emerge un sujeto dominante.

En segundo lugar, se trató de determinar hasta qué punto el mero hecho de ser una minoría numérica facilita la dominancia del individuo. 


\section{METODO}

Sujetos: se citó a 32 sujetos, nueve de los cuales se retiraron en un momento u otro del período de observación, quedando pues 23 . Se trataba de mujeres, estudiantes de los primeros cursos de Universidad distribuidas al azar en seis grupos de cuatro miembros (excepto un grupo que fue de tres). Se cuidó explícitamente que los sujetos no se conocieran entre sí para evitar cualquier historia de interacción previa. La selección de los sujetos fue aleatoria en base a las listas de Secretaría.

Procedimiento: el experimento siguió la pauta general comentada anteriormente (observación previa, tratamiento y posterior observación de los sujetos del grupo).

Las dos notas más características de este experimento fueron las condiciones en las que se administró el tratamiento experimental y el tipo de tarea asignada al grupo.

Con respecto al tratamiento experimental, ya hemos mencionado que el experimentador puede manipular tres importantes variables: pertenencia a mayoría o minoría, atribución de competencia y grado de ambigüedad en la tarea.

En este caso se creó una minoría de un sujeto (siempre el sujeto 4) frente a una mayoría de tres. Las primeras tres series de tarjetas fueron las ya descritas en el procedimiento general. La cuarta serie implicó una situación perceptiva ambigua tanto para la mayoría como para la minoría. Para la mayoría la situación ambigua consistió en tres reactivos de la categoría 3, cuatro de la categoría 4 y cinco de la categoría 5 , así como uno de la categoría 7. Para la minoría la serie consistió en un reactivo de la categoría 2 , cinco de la categoría 3, cuatro de la categoría 4 y tres de la categoría 5. Por estudios anteriores (Fdez. Dols, 1981) sabemos que en tal situación es posible esperar un margen de divergencia que oscila entre cero y once (mínimo juicio: cero perros; máximo: once).

La razón para escoger dos series críticas de reactivos ambiguos fue la efectividàd de la incertidumbre en dicha situación (Fdez. Dols, 1984) para provocar una situación inesperada, difícil de interpretar en términos de la experiencia previa y en la que resulta fácil justificar diferencias en términos de competencia.

La única característica del tratamiento que no era común para mayoría y minoría fue la designación de la minoría percibida como competente: en cuatro casos el experimentador asignaba - durante el segundo y tercer ensayos- la solución a la minoría numérica; en dos casos a la mayoría (vid. la descripción del procedimiento general).

De acuerdo con esto, podemos caracterizar al diseño como un Diseño de Grupo Control con Tratamiento Inverso y Pretest/Post-test (Cook y Campbell, 1979). En la notación de Campbell y Stanley sería $₫ O \mathrm{X}$ O» vs. $\varangle \mathrm{O} X-\mathrm{O}$, donde «O» es la observación del trabajo de grupo, «X» el tratamiento que atribuye competencia a la mayoría y $« X \rightarrow$ la situación inversa, i.e., una minoría numérica a la que se le atribuye competencia.

Con respecto a la tarea del grupo, se ideó un procedimiento que permite establecer un indicador de dominancia individual de forma rápida y carente de ambigüedad.

Dicha tarea post-experimental consistía en construir una figura con siete piezas de un puzzle de tipo Tangram.

El Tangram es un puzzle de origen chino que permite la construcción de un 
número prácticamente infinito de figuras caprichosas a partir siempre de siete figuras geométricas.

Se seleccionaron cuatro figuras (un barco, un hombre, un pájaro y un gato) consideradas de dificultad similar por parte de un equipo de jueces. Los 24 sujetos fueron adiestrados unos días antes del experimento en la realización de una de las figuras de modo que, una vez en grupo, cada sujeto era capaz de llevar a cabo una figura distinta a la de los demás. Por supuesto, los sujetos no conocían esta circunstancia y creían que todos estaban entrenados para la misma figura; tampoco conocían la relación entre el adiestramiento y el experimento. Se comprobó momentos antes de la sesión que los sujetos recordaban correctamente la construcción de la figura que se les había asignado.

El propósito de esta tarea era observar si un sujeto imponía su solución unilateralmente a los otros.

La tarea pre-experimental consistía en otro puzzle de distinto tipo; en este caso los sujetos debían construir un polígono con un gran número de piezas pero todo el mundo podía participar en la actividad simultánea y cooperativamente.

Esta tarea permitía, pues, verificar, sin ambigüedad, si un miembro del grupo impone una determinado solución ya que durante la sesión los sujetos debían resolver el puzzle una sola vez y con un único conjunto de siete piezas.

Es preciso indicar que, aunque el Diseño de Grupo de Control con Tratamiento Reversible plantea algunos problemas (vid. Cook y Campbell, 1979), las dificultades para reclutar sujetos, enseñarles a resolver el Tangram y mantenerlos aislados entre sí, hizo imposible la utilización de un tercer grupo de control sin tratamiento.

\section{RESULTADOS}

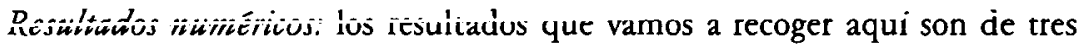
tipos: evolución de los juicios de la mayoría y la minorí? a través del tratamien-

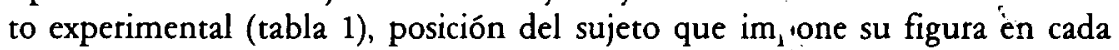
grupo y elecciones sociométricas en la entrevista post-experimental (tabla 2).

TABLA I

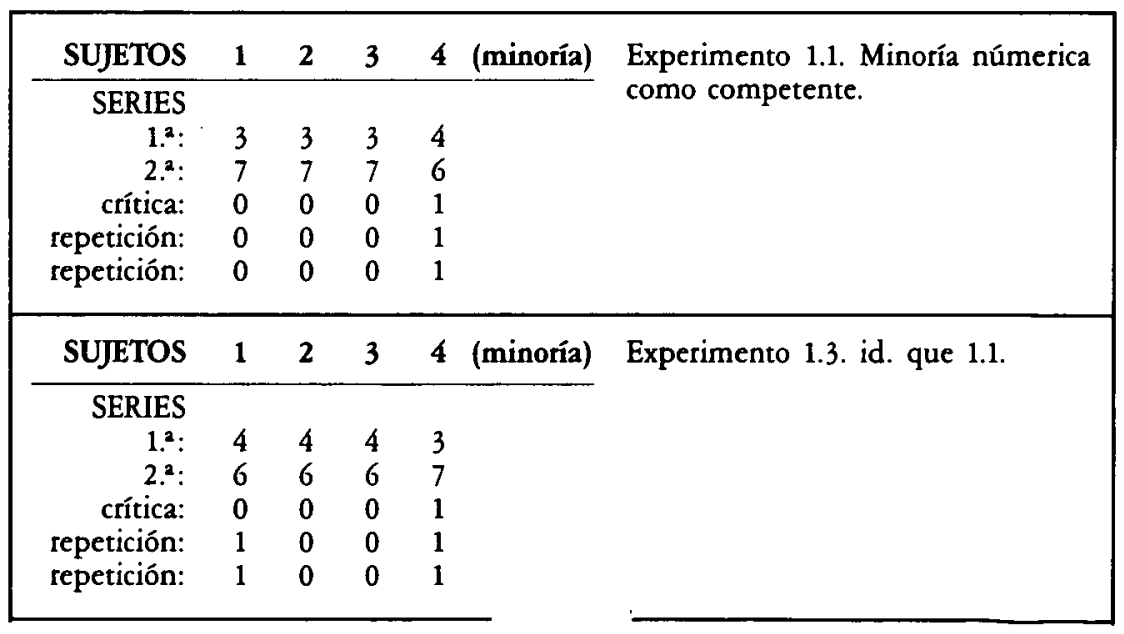




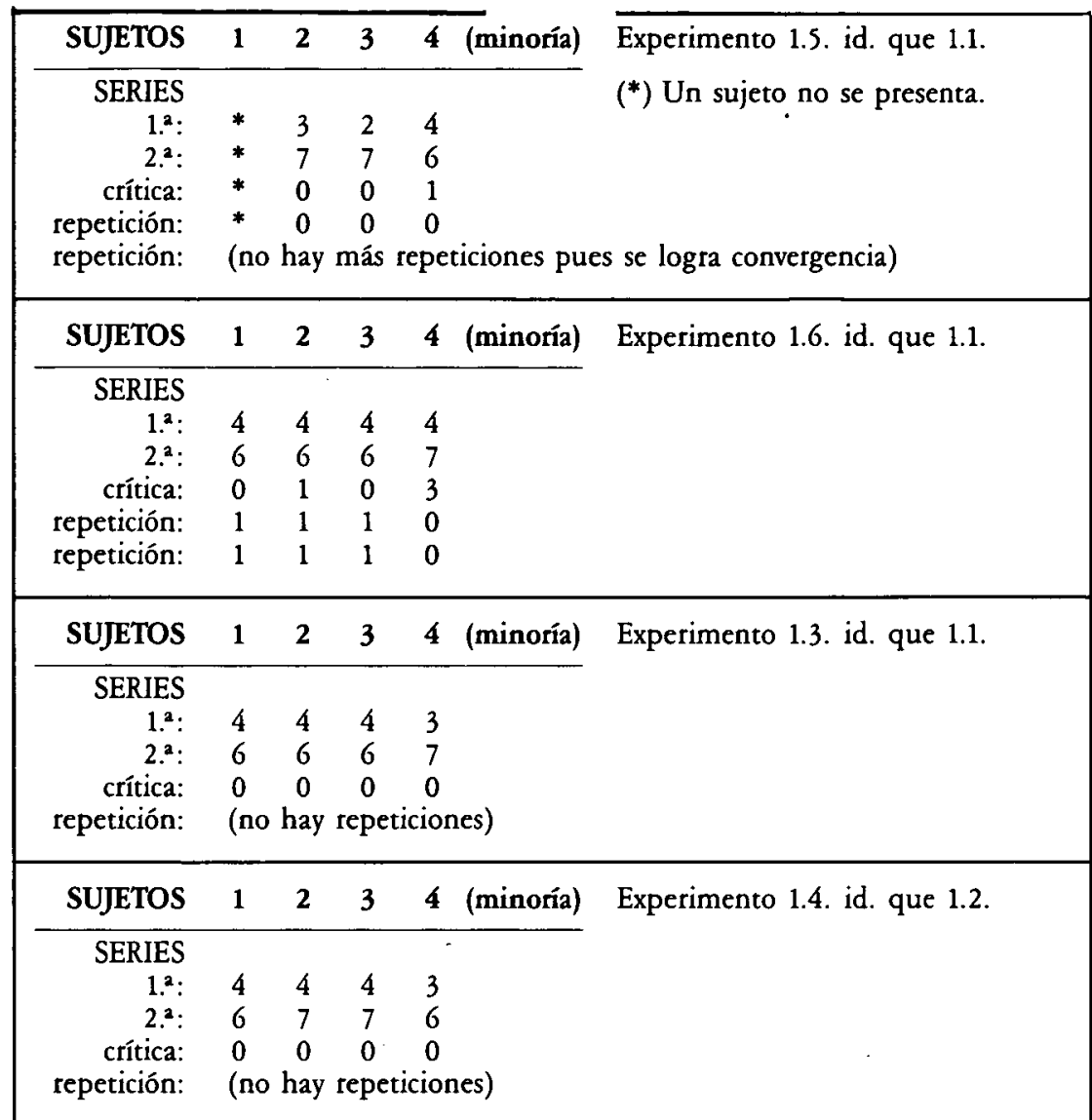

Comenzando por las figuras elaboradas en las situaciones post-tratamiento, podemos decir que en ningún caso los sujetos se pusieron de acuerdo para llevar a cabo una figura colectiva que no supusiera ninguna de las soluciones individuales. Siempre se observó una solución unilateral y los sujetos minoritarios impusieron su solución en las series 1.1, 1.4. y 1.6. (gato, pájaro y gato respectivamente) mientras que el sujeto 3 impuso la suya en la serie 1.2. (barco) y el sujeto 2 en la serie 1.5. (gato).

TABLA II

Elecciones sociométricas

EXP. 1.1.

EXP. 1.2 .

EXP. 1.3 .

\begin{tabular}{|c|c|c|c|c|c|c|c|c|c|c|c|c|c|c|}
\hline Sujetos & 1 & 2 & 3 & 4 & Sujetos & 1 & 2 & 3 & 4 & Sujetos & 1 & 2 & 3 & 4 \\
\hline 1 & 0 & 0 & 0 & 1 & 1 & 0 & 0 & 1 & 0 & 1 & 0 & 0 & 0 & 1 \\
\hline 2 & 0 & 0 & 0 & 1 & 2 & 0 & 0 & 0 & 0 & 2 & 1 & 0 & 0 & 0 \\
\hline 3 & 0 & 1 & 0 & 0 & 3 & 0 & 0 & 0 & 1 & 3 & 0 & 1 & 0 & 0 \\
\hline 4 & 0 & 0 & 1 & 0 & 4 & 1 & 0 & 0 & 0 & 4 & 1 & 0 & 0 & 0 \\
\hline
\end{tabular}




\begin{tabular}{|c|c|c|c|c|c|c|c|c|c|c|c|c|c|c|}
\hline $\begin{array}{l}\text { EXP. } 1.4 . \\
\text { Sujetos }\end{array}$ & 1 & 2 & 3 & 4 & $\begin{array}{l}\text { EXP. 1.5. } \\
\text { Sujetos }\end{array}$ & 1 & 2 & 3 & 4 & $\begin{array}{l}\text { EXP. 1.6. } \\
\text { Sujetos }\end{array}$ & 1 & 2 & 3 & 4 \\
\hline 1 & 0 & 1 & 0 & 0 & 1 & 0 & 0 & & 0 & 1 & 0 & 0 & 0 & 0 \\
\hline 2 & 0 & 0 & 0 & 1 & 2 & 0 & 0 & & 1 & 2 & 0 & 0 & 0 & 1 \\
\hline 3 & 0 & 0 & 0 & 1 & 3 & & & & & 3 & 0 & 0 & 0 & 1 \\
\hline 4 & 0 & 1 & 0 & 0 & 4 & 0 & 0 & & 0 & 4 & 0 & 0 & 1 & 0 \\
\hline
\end{tabular}

En lo que se refiere a las matrices sociométricas (vid. tabla 2), consideradas como un todo, la minoría obtiene nueve elecciones, los sujetos en la posición 1 de la mayoría tres, los sujetos en posición 2 de la mayoría cuatro y los sujetos en la posición 3 de la mayoría tres.

Efectos del experimento en conformidad: los efectos del tratamiento experimental en la convergencia de mayoría y minoría coinciden con el patrón general establecido previamente en la taxonomía de Fernández Dols et al. (1984). En cada caso se produce una relativa convergencia de mayoría y minoría y, en tres de ellos, una absoluta convergencia de la minoría. En los dos casos en los que la mayoría numérica recibe la atribución de competencia, la conformidad minoritaria aparece de manera automática tras la primera exposición de la serie crítica de reactivos.

Por otra parte, la minoría «competente» no parece provocar una especial con: fluencia de los sujetos mayoritarios. Parece la minoría la empeñada en hacer el mayor esfuerzo por converger.

Observación Post-tratamiento: Los resultados de la tarea post-tratamiento y de las elecciones sociométricas (indicadores de dominancia) fueron relativamente independientes de la dirección de la influencia social y más favorables para los sujetos minoritarios.

En primer lugar, y como ya se indicó, no hay trabajo cooperativo: siempre

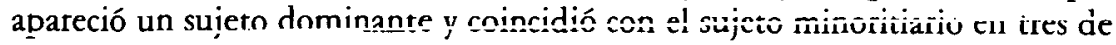
las seis ocasiones.

En términos estadísticos, si suponemos que todos los sujetos tienen la misma probabilidad de ser «dominantes» (imponer su figura), la probabilidad, de acuerdo con el Test Binomial para una muestra, de que los sujetos de la mayoría impongan su solución en tres o menos casos es inferior a $.17(\mathrm{n}=6 ; \mathrm{p}=.75)$; sin embargo, si partimos de una hipótesis nula, según la cual la minoría puede ser dominante sólo en los casos en los que no se conforma, el Test Binomial ( $\mathbf{n}=6 ; \mathbf{p}=.85$ ) muestra que la probabilidad asociada al hecho de que la mayoría imponga su solución en tres casos o menos es .04 .

Con respecto a las elecciones sociométricas, debemos tener en cuenta que los sujetos no se conocían entre sí antes del experimento; sin embargo, los sujetos mostraron preferencias por la minoría más frecuentemente que por la mayoría. Excepto en una de las series experimentales (1.3.), en la que se prefirió a un sujeto dominante no minoritario, la minoría siempre obtiene un número de elecciones igual o superior a los más elegidos de la mayoría. Esta uniformidad en todos los casos hace improbable que tal preferencia se deba al período de interacción previo al tratamiento experimental.

Si calculamos, por medio del Test Binomial para una muestra ( $\mathrm{n}=19$; $\mathrm{p}=.74$ ), la probabilidad asociada al hecho de que sólo 10 o menos de las 19 elecciones llevadas a cabo fueran favorables a los 17 sujetos mayoritarios, esta probabilidad es .045 . 
Problemas planteados: Obviamente, aun cuando no podemos establecer conclusiones definitivas respecto al efecto de la posición minoritaria en las relaciones de dominancia del grupo, un hecho es cierto: hay una clara tendencia de la minoría a «dominan en la tarea y en las preferencias afectivas del grupo, independientemente del grado de sinfluencias mayoritaria o minoritaria que se manifieste en un momento anterior de la vida del grupo.

También existe una clara relación en las elecciones sociométricas a la minoría: estas elecciones parecen tener lugar cuando la minoría ha impuesto su figura. La tabla 2 muestra que la minoría es preferida precisamente en las series experimentales en las que «dominas en la tarea (1.1.; 1.4. y 1.6.).

Estos resultados son muy sugerentes y parecen confirmar un efecto de dominancia afectiva y conductual de la minoría. La relación entre competencia percibida y dominancia parece estar, sobre todo, mediada por la posición del sujeto en el grupo y no tanto por el grado de influencia o conformidad manifestados en la tarea perceptiva. A este respecto, la minoría parece tener ciertas ventajas sobre el resto de los individuos del grupo para establecer una relación de dominancia.

Todos estos hallazgos hacen necesario un segundo experimento que, desde un diseño similar, modifique las condiciones en el tratamiento experimental y las tareas pre y post tratamiento, con vistas a verificar estas observaciones.

\section{EXPERIMENTO 2}

\section{METODO}

Sujetos: 24 estudiantes, de sexo femenino, que no habían interactuado previamente entre sí, seleccionadas al azar entre las personas que acudían al comedor univesitario.

Procedimiento: el procedimiento general para este experimento es el mismo que en el caso del experimento 1; hay, sin embargo, variaciones en el tipo de tareas pre y post-tratamiento y en las variables manipuladas en dicho tratamiento.

El propósito del experimento fue estudiar la pauta de dominancia minorita- ria en un contexto cooperativo y ambiguo para observar si, en tales circunstancias, la minoría seguía mostrando una mayor tendencia a la dominancia que la mayoría.

Con vistas a lograr dichos propósitos, se intentó repetir el diseño del experimento 1 (OXO vs. OX-O), lo que nos permitiría comparar los resultados de ambos experimentos. Sin embargo, en este caso se trató de lograr unas relaciones de influencia mayoritaria y minoritaria más marcadas para comprobar el grado de interdependencia de dichos fenómenos con respecto al de dominancia minoritaria.

Así, en un caso la mayoría numérica fue la que recibió el apoyo del experimentador en los ensayos divergentes pero encontrándose además en una situaçión perceptiva carente de ambigüedad, mientras que la minoría no sólo no recibía atribución de competencia sino que además se encontraba en una situación perceptiva ambigua. En las series experimentales del grupo con tratamiento inverso fue la minoría la que se encontró en situación perceptiva carente de ambigüedad frente a una mayoría en condiciones opuestas.

Las diferencias en el grado de ambigüedad del estímulo en la tarea perceptiva se obtuvieron mostrando por una parte de las tarjetas algunas viñetas con 
dibujos claramente definidos (en la escala gráfica de Frenkel-Brunswik: siete gatos y ocho perros) y por la otra una condición muy diferente: dos figuras claras y trece ambiguas (vid. el § Metodología General).

«OXO» es una situación en la que la minoría numérica comete, según el experimentador, juicios erróneos y sufre un marco perceptivo ambiguo al que la mayoría no está expuesta. Según nuestra investigación anterior y la hipótesis de este trabajo, se observarán (más claramente que en el experimento 1) comportamientos conformistas de la minoría. Además, la atribución de competencia a la mayoría facilitará la dominancia de la mayoría aunque la dominancia de cualquiera de los sujetos mayoritarios deberá ser muy inferior a la observada a la minoría en $₫ \mathrm{OX}-\mathrm{O}$.

«OX-O» es una situación en la que la minoría numérica parece ser la que hace los juicios correctos y se enfrenta a una mayoría expuesta a un marco perceptivo ambiguo. En este caso podrá aparecer una débil influencia minoritaria en la tarea perceptiva pero, sobre todo, un claro patrón de dominancia por parte de la minoría en la interacción del grupo.

Tarea: en este experimento 2, los sujetos trabajaron en grupo antes y después del tratamiento en un juego cooperativo, en concreto construir una carretera de juguete o un pueblo en una mesa, utilizando materiales del Test del Pueblo.

La dominancia de los sujetos se estableció aquí de un modo menos artificioso que en el experimento 1: el experimentador indicaba la tarea y, una vez acaba$\mathrm{da}$ (sentado todavía de forma aparentemente casual de cara a la pared evitando toda interacción visual o facial con los sujetos) les solicitaba que explicaran lo que habían hecho.

A partir de los datos registrados por vídeo se observó quien había actuado como portavoz de los miembros del grupo. Para ello se llevó a cabo una observación sistemática de la secuencia a intervalos de cinco segundos, registrándose diversas conductas verbales y no verbales de los sujetos.

De todos estos registros había uno que indicaba quién dirigía la exposición por parte de los miembros del grupo: el habla acompañada por «ilustradores» (según la terminología de Ekman y Friesen, 1969) destinados a dirigir la atención (deicticos o conductas de punteo). Se consideró la frecuencia de emisión de punteos como criterio más objetivo para detectar el habla acompañada por dirección de la atención del grupo.

La conducta de punteo fue registrada en vídeo, analizándose la secuencia que comenzaba cuando el experimentador solicitaba al grupo que explicara lo que habían hecho y finalizaba cuando los sujetos concluían la explicación. Dicha secuencia se descompuso en intervalos de cinco segundos. Dos observadores entrenados registraban la presencia o ausencia de punteo en la conducta verbal de los sujetos del grupo.

\section{RESULTADOS}

Resultados numéricos: los resultados numéricos que se muestran en la tabla III se refieren a la evolución de los juicios de la mayoría y la minoría con respecto al número de perros percibido. La tabla IV muestra las elecciones sociométricas recibidas por los sujetos en la entrevista post-experimental. La tabla V se refiere al número de intervalos en los que cada sujeto exhibe punteo dividido por el total de punteo en su grupo (se indica además el número total de intervalos observados en cada grupo). 
Efectos del tratamiento en conformidad inmediata: debido al tipo de manipulación de variables utilizada en este experimento 2, los resultados muestran un efecto claramente divergente, incluso inverso, de los dos grupos entre sí. En el caso de los grupos en los que la minoría está en las condiciones más adver. sas (OXO) aparece conducta conformista en dos de los tres grupos. Debemos señalar que, en el caso de la serie experimental 2.3. la entrevista post-experimental reveló que el sujeto minoritario sospechaba algún engaño; de hecho es llamativa la brusca divergencia del sujeto minoritario durante la repetición de los ensayos críticos (probablemente a partir del punto en el que sintió sospechas).

TABLA III

\begin{tabular}{|c|c|c|c|c|c|c|}
\hline SUJETOS & 1 & 2 & 3 & 4 & (minoría) & \multirow{2}{*}{$\begin{array}{l}\text { Experimento 2.1. Mayoría númerica } \\
\text { competente. }\end{array}$} \\
\hline $\begin{array}{r}\text { SERIES } \\
1 .^{2}: \\
2 .^{2}: \\
\text { crítica: } \\
\text { repetición: } \\
\text { repetición: }\end{array}$ & $\begin{array}{l}3 \\
7 \\
8\end{array}$ & $\begin{array}{l}3 \\
7 \\
8\end{array}$ & $\begin{array}{l}3 \\
7 \\
8\end{array}$ & $\begin{array}{l}4 \\
7 \\
8\end{array}$ & & \\
\hline SUJETOS & 1 & 2 & 3 & 4 & (minoria) & \multirow[t]{2}{*}{ Experimento 2.2. id. que 2.1. } \\
\hline $\begin{array}{r}\text { SERIES } \\
1 .^{\mathrm{a}} \text { : } \\
2 .^{2}: \\
\text { crítica: } \\
\text { repetición: } \\
\text { repetición: }\end{array}$ & $\begin{array}{l}4 \\
6 \\
8 \\
8 \\
8\end{array}$ & $\begin{array}{l}4 \\
6 \\
8 \\
8 \\
8\end{array}$ & $\begin{array}{l}4 \\
6 \\
8 \\
8 \\
8\end{array}$ & $\begin{array}{l}3 \\
6 \\
1 \\
8 \\
9\end{array}$ & & \\
\hline SUJETOS & 1 & 2 & 3 & 4 & (minoría) & \multirow[t]{2}{*}{ Experimento 2.3. id. que 2.1} \\
\hline $\begin{array}{r}\text { SERIES } \\
1 .^{2}: \\
2 .^{2}: \\
\text { crítica: } \\
\text { repetición: } \\
\text { repetición: }\end{array}$ & $\begin{array}{l}3 \\
5 \\
8 \\
8 \\
8\end{array}$ & $\begin{array}{l}4 \\
6 \\
8 \\
8 \\
8\end{array}$ & $\begin{array}{l}4 \\
6 \\
8 \\
8 \\
8\end{array}$ & $\begin{array}{l}3 \\
7 \\
7 \\
1 \\
1\end{array}$ & & \\
\hline $\begin{array}{r}\text { SUJETOS } \\
\text { SERIES } \\
1 .^{2}: \\
2 .^{2}: \\
\text { crítica: } \\
\text { repetición: } \\
\text { repetición: }\end{array}$ & $\begin{array}{l}4 \\
7 \\
3 \\
1 \\
1\end{array}$ & $\begin{array}{c}4 \\
7 \\
7 \\
10 \\
14\end{array}$ & $\begin{array}{l}4 \\
7 \\
1 \\
1 \\
1\end{array}$ & $\begin{array}{l}3 \\
6 \\
8 \\
8 \\
8\end{array}$ & (minoría) & $\begin{array}{l}\text { Experimento 2.4. Tratamiento inver- } \\
\text { so; Minoría numérica competente. }\end{array}$ \\
\hline SUJETOS & 1 & 2 & 3 & 4 & (minoría) & \multirow[t]{2}{*}{ Experimento 2.5 . id. que 2.4 . } \\
\hline $\begin{array}{r}\text { SERIES } \\
1 .^{\mathrm{a}}: \\
2 .^{2}: \\
\text { crítica: } \\
\text { repetición: } \\
\text { repetición: }\end{array}$ & $\begin{array}{l}6 \\
6 \\
1 \\
1 \\
2\end{array}$ & $\begin{array}{l}4 \\
6 \\
2 \\
2 \\
2\end{array}$ & $\begin{array}{c}4 \\
6 \\
10 \\
9 \\
9\end{array}$ & $\begin{array}{l}3 \\
7 \\
8 \\
8 \\
8\end{array}$ & & \\
\hline
\end{tabular}




\begin{tabular}{|c|c|c|c|c|c|}
\hline SUJETOS & 1 & 2 & 3 & 4 (minoría) & Experimento 2.6. id. que 2.4 . \\
\hline \multicolumn{6}{|l|}{ SERIES } \\
\hline 1. ${ }^{2}:$ & 4 & 4 & 4 & 3 & \\
\hline 2.2: & 6 & 6 & 5 & 7 & \\
\hline crítica: & 6 & 7 & 7 & 8 & \\
\hline repetición: & 10 & 8 & 4 & 8 & \\
\hline repetición & 8 & 9 & 7 & 8 & \\
\hline
\end{tabular}

En el grupo con tratamiento simétrico, la minoría no se somete a los juicios de la mayoría y en un caso específico (serie experimental 2.6.) produce una cierta convergencia de los juicios de la mayoría hacia los propios.

En el presente informe no nos interesa extendernos sobre el problema de la conformidad en estos grupos. Nuestro equipo ha llevado previamente diversos estudios específicos sobre este tema con diseños factoriales más completos (vid. Fernández Dols, 1981; Fernández Dols et al., 1984). Lo que nos interesa aquí es constatar que, incluso con un tratamiento experimental que provoca claros fenómenos de influencia social mayoritaria y minoritaria en el grupo, no existe una interdependencia entre dichos fenómenos y las conductas de dominancia.

Observación post-tratamiento: si se consultan las tablas correspondientes nos encontramos aquí de nuevo con los datos más significativos (vid. tabla $\mathrm{V}$ ). La única parte de la tabla $\mathrm{V}$ en la que la conducta de punteo aparece sistemáticamente y en una proporción significativa, es la que se refiere a la sesión posttratamiento de las series experimentales 2.4., 2.5. y 2.6., es decir, aquéllas en las que la minoría es «competente» y la mayoría no (OX-O).

En la condición OXO no hay dominancia minoritaria pero la dominancia de los sujetos mayoritarios aparece de forma más débil (hay conducta de punteo en sólo uno de los grupos OXO y éste está muy disperso); este dato coincidè

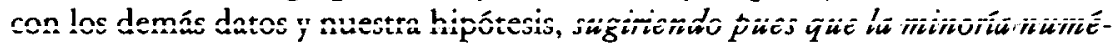
rica tiene en su misma condición un factor facilitador de su dominancia que no tiene la mayoría y que no existe una relación directa entre influencia social $y$ dominancia.

Si aplicamos un ANOVA con una transformación previa de los datos (Arc Sen $\sqrt{p}$ ) que aparecen en la tabla $V$ detectamos un efecto significativo'y muy fuerte en las diferencias entre mayoría y minoría para las observaciones posttratamiento de la condición $\mathrm{OX}-\mathrm{O}(\mathrm{F}=14.73 ; \mathrm{p}<.01)$, y, sobre todo, en la interacción «pertenencia a mayoría vs. minoría $\mathrm{X}$ condición experimental OXO vs. OX-O» $(\mathrm{F}=33.53 ; \mathrm{p}<.001)$. Así pues la dirección de la atención de la minoría mediante el punteo en la condición OX-O es muy notoria. Los ANOVA para las observaciones pre-tratamiento no fueron significativas en ningún caso, lo que nos permite desechar la existencia de diferencias previas al tratamiento entre los grupos.

Además, podemos ver que la tendencia, ya observada en los experimentos previos, según la cual la minoría es más popular que la mayoría, se mantiene también aquí a través de las dos condiciones del diseño. Si por medio del Test Binomial para una muestra calculamos la probabilidad asociada al hecho de que 16 o menos, de las 26 elecciones hechas, sean favorables a los 18 sujetos en situación mayoritaria (frente a 10 elecciones para los 6 minoritarios), dicha probabilidad está por debajo de .20. Sin embargo, hay una importante influencia del tratamiento en estos resultados: en OXO la probabilidad asociada al hecho 
de que la mayoría obtenga 11 elecciones o menos (de las 16 elecciones hechas) es aproximadamente .40 , mientras que en la segunda condición (OX-O; competencia de la minoría) es .08 .

Según todo esto, es posible postular un efecto de dominancia afectiva y conductual de la minoria; la competencia percibida parece tener un efecto facilitador de dicha dominancia en el caso de la minoría pero no en el de la mayoría.

\section{CONCLUSIONES}

Los resultados obtenidos de nuestros experimentos parecen demostrar la viabilidad de nuestra hipótesis. Parece que la aparición de la dominancia no guarda una relación directa con la influencia social y que se da más fácilmente en los sujetos minoritarios. Ello, más que refutar otras perspectivas sobre las minorías en grupos parece complementarlas.

Así, por ejemplo, la actual investigación sobre la influencia minoritaria plantea una controversia sobre si la influencia de la mayoría y de la minoría corresponde al mismo proceso básico (Wolf, 1985).

La distinción entre influencia minoritaria y dominancia minoritaria no resuelve el problema pero proporciona una cierta clarificación conceptual ya que la dominancia minoritaria podría implicar un proceso similar que, en el caso de la mayoría, el fenómeno por el que la minoría se pliega a la mayoría sólo externamente.

Así, hemos visto que la competencia percibida de la minoría y posiblemente otras variables (como por ejemplo certidumbre perceptiva) facilitan su dominancia. En el caso de la mayoría numérica la competencia percibida y la certidumbre perceptiva parecen facilitar su influencia sobre la minoría pero no su dominancia. ¿Se trata de dos aspectos de un mismo fenómeno apareciendo en diferentes momentos de la interacción del grupo?

Si ello fuera cierto, podríamos suponer que existirían dos continuos: en uno se establecería la oposición entre dominancia minoritaria y conformidad, que se produce en términos fundamentalmente conductuales y afectivos, mientras que el otro continuo recoge los polos conversión vs. normalización, y sería fundamentalmente perceptivo y cognitivo.

En conclusión, la confrontación entre mayoría y minoría descansa en un proceso no sólo de influencia sino también de dominancia social que debemos comenzar a analizar a un nivel interactivo y afectivo. Una de las hipótesis más razonables de nuestra investigación es que el proceso subyacente descansa en una orientación social de la atención, un fenómeno que ha sido estudiado por Chance (1967) en Etología, con respecto a las relaciones de dominancia y por Taylor y Fiske (1978) en Psicología Social con respecto al estudio de la saliencia; Fiske y Taylor (1984) plantean explícitamente la relación entre saliencia y dominancia. 


\section{1}

TABLA IV

Elecciones sociométricas (segunda serie experimental)

EXP. 2.1 .

EXP. 2.2.

EXP. 2.3.

\begin{tabular}{rrrrr} 
Sujetos & 1 & 2 & 3 & 4 \\
\hline 1 & 0 & 1 & 1 & 1
\end{tabular}

\begin{tabular}{rllll} 
Sujetos & 1 & $\mathbf{2}$ & $\mathbf{3}$ & $\mathbf{4}$ \\
\hline 1 & 0 & 0 & 0 & 1
\end{tabular}

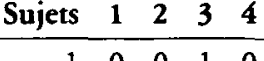

$\begin{array}{lllll}2 & 1 & 0 & 1 & 1\end{array}$

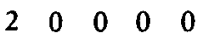

$3 \quad 1 \quad 0000$

$3 \quad 0 \quad 00001$

$\begin{array}{llllll}4 & 0 & 0 & 0 & 0\end{array}$

$\begin{array}{lllll}4 & 0 & 0 & 1 & 0\end{array}$

$\begin{array}{lllll}2 & 0 & 0 & 0 & 1 \\ 3 & 1 & 0 & 0 & 0 \\ 4 & 1 & 1 & 1 & 0\end{array}$

EXP. 2.4.

EXP. 2.5.

EXP. 2.6.

\begin{tabular}{rrrrr} 
Sujetos & 1 & 2 & 3 & 4 \\
\hline 1 & 0 & 0 & 0 & 0 \\
2 & 1 & 0 & 0 & 0 \\
3 & 0 & 0 & 0 & 1 \\
4 & 0 & 0 & 0 & 0
\end{tabular}

\begin{tabular}{rrrrr} 
Sujetos & $\mathbf{1}$ & $\mathbf{2}$ & $\mathbf{3}$ & $\mathbf{4}$ \\
\hline 1 & 0 & 0 & 1 & 0 \\
2 & 0 & 0 & 1 & 0 \\
3 & 0 & 0 & 0 & 1 \\
4 & 0 & 1 & 0 & 0
\end{tabular}

Sujeto

$\begin{array}{lllll}1 & 0 & 0 & 0 & 1 \\ 2 & 0 & 0 & 0 & 1 \\ 3 & 0 & 0 & 0 & 1 \\ 4 & 0 & 1 & 0 & 0\end{array}$

TABLA $\mathrm{V}$

Punteos del sujeto divididos por el total de punteos emitidos en el grupo

\begin{tabular}{|ccccccccc|}
\hline SUJETOS & \multicolumn{2}{c}{${ }^{1.2}$} & \multicolumn{2}{c}{ 2. $^{2}$} & \multicolumn{2}{c|}{$3^{2}$} & \multicolumn{2}{c|}{ minoría } \\
& PRE. & POST. & PRE. & POST. & PRE. & POST. & PRE. & POST. \\
\hline & $(\mathrm{a})$ & $(\mathrm{b})$ & $(\mathrm{a})$ & $(\mathrm{b})$ & $(\mathrm{a})$ & $(\mathrm{b})$ & $(\mathrm{a})$ & $(\mathrm{b})$ \\
exper. 2.1:: & $1 / 4$ & $3 / 6$ & $3 / 4$ & $1 / 6$ & $0 / 4$ & $2 / 6$ & $0 / 4$ & $0 / 6$ \\
\hline & $(\mathrm{c})$ & $(\mathrm{d})$ & $(\mathrm{c})$ & $(\mathrm{d})$ & $(\mathrm{c})$ & $(\mathrm{d})$ & $(\mathrm{c})$ & $(\mathrm{d})$ \\
exper. 2.2.: & $0 / 4$ & $0 / 0$ & $2 / 4$ & $0 / 0$ & $1 / 4$ & $0 / 0$ & $1 / 4$ & $0 / 0$ \\
\hline & $(\mathrm{e})$ & $(\mathrm{f})$ & $(\mathrm{e})$ & $(\mathrm{f})$ & $(\mathrm{e})$ & $(\mathrm{f})$ & $(\mathrm{e})$ & $(\mathrm{f})$ \\
exper. 2.3.: & $0 / 1$ & $0 / 0$ & $0 / 1$ & $0 / 0$ & $0 / 1$ & $0 / 0$ & $1 / 1$ & $0 / 0$ \\
\hline & $(\mathrm{g})$ & $(\mathrm{h})$ & $(\mathrm{g})$ & $(\mathrm{h})$ & $(\mathrm{g})$ & $(\mathrm{h})$ & $(\mathrm{g})$ & $(\mathrm{h})$ \\
exper. 2.4.: & 0 & $0 / 2$ & 0 & $0 / 2$ & 0 & $0 / 2$ & 0 & $2 / 2$ \\
\hline & $(\mathrm{i})$ & $(\mathrm{j})$ & $(\mathrm{i})$ & $(\mathrm{j})$ & $(\mathrm{i})$ & $(\mathrm{j})$ & $(\mathrm{i})$ & $(\mathrm{j})$ \\
exper. 2.5.: & $0 / 0$ & $0 / 6$ & $0 / 0$ & $0 / 6$ & $0 / 0$ & $1 / 6$ & $0 / 0$ & $5 / 6$ \\
\hline & $(\mathrm{k})$ & $(\mathrm{l})$ & $(\mathrm{k})$ & $(\mathrm{l})$ & $(\mathrm{k})$ & $(\mathrm{l})$ & $(\mathrm{k})$ & $(\mathrm{l})$ \\
exper. 2.6.: & $0 / 4$ & $0 / 5$ & $0 / 4$ & $0 / 5$ & $1 / 4$ & $0 / 5$ & $3 / 4$ & $5 / 5$ \\
\hline
\end{tabular}

(a) Número total de intervalos observados $=10$

(b) Número total de intervalos observados $=14$

(c) Número total de intervalos observados $=15$

(d) Número total de intervalos observados $=15$

(e) Número total de intervalos observados $=21$

(f) Número total de intervalos observados $=10$

(g) Número total de intervalos observados $=$ los sujetos no hablan tras la tarea.

(h) Número total de intervalos observados $=2$

(i) Número total de intervalos observados $=9$

(j) Número total de intervalos observados $=9$

(k) Número total de intervalos observados $=17$

(l) Número total de intervalos observados $=11$ 


\section{Referencias}

CAMPBelL, D.T., y StanLEY. J.C. (1966). Experimental and Quasi-Experimental Designs for Research. Rand McNally, Chicago.

COOK, T.D., y CAMPBELL, D.T. (1979). Quasi-Experimentation. Design and Analysis Issues for Field Settings, Houghton Mifflin, Boston.

CHANCE, M.R.A. (1967). Attention Structure as the Basis of Primate Rank Orderso. Man, 2:503-518.

CHANCE, M.R.A., y LARSEN, R.R. (eds.). (1976). The Social Structure of Attention, Wiley, New York.

DE Wall, F. (1982). Chimpance Politics, Harper and Row, New York.

EKMAN, P., y FriesEN, W.V. (1969). The Repertoire of Non-verbal Behavior: Categories, Origins, Usage and Coding*. Semiotica, 1:49-98.

FERNÁNDEZ-DOLS. J.M. (1981). El estudio psicológico del orden social: una elaboración experimental a partir del estudio de la conformidad en Asch y Sherif. Editorial de la Universidad Complutense, Madrid.

FernÁNDEZ-Dols, J.M.; Corraliza, J.A.; ReVERTER, E., y Movellán, J. (1984). «Influencia social e incertidumbre: una taxonomia experimentals. Revista de Psicología General y Aplicada, 39:905-940.

FISKE, S.T., y TAYLOR, S.E. (1984). Social Cognition. Addison-Wesley, Reading Mass.

Frenkel-Brunswik, E. (1948). A Study of Prejudice in Childrens. Human Relations, 1:295-306.

GOITMAN, J., y RINGLAND, J. (1981). The analysis of dominance and bidirectionality in social developments. Child Development, 52:393-412.

MoscovicI, S. (1976). Social Influence and Social Change. Academic, London.

MoscovicI, S. (1985). Social Influence and Conformitys. En: Lindzey, G., y Aronson, E. (eds.). Handbook of Social Psychology. Random House, New York.

PELÁEZ, F. (1985). «La dominancia social: una aproximación primatológicar. Estudios de Psicologia, 21:99-113.

RICHARDS, S.M. (1974). aThe Concept of Dominance and Methods of Assesments. Animal Behavior, 22:914-930.

ROWELL, T.E. (1974). The Concept of Social Dominances. Behavioral Biology, 11:131-154.

SHAw, M.E. (1976). Group Dynamics. McGraw-Hill, New York.

SwCKIN, A.M.; SMITH, P.K. (1977). Two approaches to the concept of dominance in preschool childrens. Child Development, 48:917-923.

STRAYER, F.F., y STRAYER, J. (1976). «An ethological analysis of social agonism and dominance relations among preschool childrens. Child Development, 47:980-989.

TAYLOR, S.E., Y FISKE, S.T. (1978). salience, attention and attribution: Top of the head phenomenas. In: Berkowitz, L. (ed.). Advances in Experimental Social Psychology (vol. 11). Academic, New York.

WOLF S. (1985). «Manifest and Latent Influences of Majorities and Minoritiess. Joumal of Personality and Social Psychology, 48:899-909. 